\title{
Protective effect of Growth Hormone-Releasing Hormone agonist in bacterial toxin-induced pulmonary barrier dysfunction
}

\author{
Istvan Czikora ${ }^{1}$, Supriya Sridhar ${ }^{1}$, Boris Gorshkov ${ }^{1}$, Irina B. Alieva ${ }^{1,2}$, Anita Kasa ${ }^{1}$, Joyce Gonzales $^{3}$, \\ Olena Potapenko ${ }^{1}$, Nagavedi S. Umapathy ${ }^{1}$, Helena Pillich ${ }^{4}$, Ferenc G. Rick ${ }^{5,6}$, Norman L. Block ${ }^{5,79,10}$, \\ Alexander D. Verin ${ }^{1}$, Trinad Chakraborty ${ }^{5}$, Michael A. Matthay ${ }^{8}$, Andrew V. Schally,7,9,10* and \\ Rudolf Lucas ${ }^{1,3,11 *}$
}

1 Department of Pharmacology and Toxicology, Vascular Biology Center, Georgia Regents University, Augusta, GA, USA

2 Department of Electron Microscopy, A.N. Belozorksy Institute, Moscow State University, Moscow, Russia

${ }^{3}$ Department of Medicine, Division of Pulmonary Medicine, Medical College of Georgia, Georgia Regents University, Augusta, GA, USA

${ }^{4}$ Department of Medicine, Institute of Medical Microbiology, Justus-Liebig University Giessen, Giessen, Germany

${ }^{5}$ Endocrine, Polypeptide and Cancer Institute, Veterans Affairs Medical Center, Miami, FL, USA

${ }^{6}$ Department of Urology, Herbert Wertheim College of Medicine, Florida International University, Miami, FL, USA

7 Department of Pathology, Miller School of Medicine, University of Miami, Miami, FL, USA

${ }^{8}$ Department of Medicine and Anesthesia, Cardiovascular Research Institute, University of California San Francisco, San Francisco, CA, USA

${ }^{9}$ Department of Medicine, Miller School of Medicine, University of Miami, Miami, FL, USA

10 Sylvester Comprehensive Cancer Center, Miller School of Medicine, University of Miami, Miami, FL, USA

"Department of Pharmacology and Toxicology, Medical College of Georgia, Georgia Regents University, Augusta, GA, USA

\section{Edited by:}

Shruti Sharma, Georgia Regents

University, USA

\section{Reviewed by:}

Deepesh Pandey, Georgia Health

Sciences University, USA

Pimonrat Ketsawatsomkron,

University of lowa, USA

\section{*Correspondence:}

Andrew V. Schally, Department of Pathology, Veterans Affairs Medical Center and South Florida Veterans Affairs Foundation for Research and Education, Miller School of Medicine, University of Miami, Miami, FL 33136, USA

e-mail: andrew.schally@va.gov; Rudolf Lucas, Division of Pulmonary and Critical Care Medicine, Department of Pharmacology and Toxicology, Vascular Biology Center, Medical College of Georgia, Georgia Regents University,

1459 Laney-Walker Blvd., Augusta, GA 30912-2300, USA

e-mail: rlucas@gru.edu
Rationale: Antibiotic treatment of patients infected with $\mathrm{G}^{-}$or $\mathrm{G}^{+}$bacteria promotes release of the toxins lipopolysaccharide (LPS) and pneumolysin (PLY) in their lungs. Growth Hormone-releasing Hormone (GHRH) agonist JI-34 protects human lung microvascular endothelial cells (HL-MVEC), expressing splice variant 1 (SV-1) of the receptor, from PLY-induced barrier dysfunction. We investigated whether JI-34 also blunts LPS-induced hyperpermeability. Since $\mathrm{GHRH}$ receptor (GHRH-R) signaling can potentially stimulate both cAMP-dependent barrier-protective pathways as well as barrier-disruptive protein kinase $\mathrm{C}$ pathways, we studied their interaction in GHRH agonist-treated HLMVEC, in the presence of PLY, by means of siRNA-mediated protein kinase A (PKA) depletion.

Methods: Barrier function measurements were done in HL-MVEC monolayers using Electrical Cell substrate Impedance Sensing (ECIS) and VE-cadherin expression by Western blotting. Capillary leak was assessed by Evans Blue dye (EBD) incorporation. Cytokine generation in broncho-alveolar lavage fluid (BALF) was measured by multiplex analysis. PKA and PKC- $\alpha$ activity were assessed by Western blotting.

Results: GHRH agonist JI-34 significantly blunts LPS-induced barrier dysfunction, at least in part by preserving VE-cadherin expression, while not affecting inflammation. In addition to activating PKA, GHRH agonist also increases PKC- $\alpha$ activity in PLY-treated HL-MVEC. Treatment with PLY significantly decreases resistance in control siRNA-treated HL-MVEC, but does so even more in PKA-depleted monolayers. Pretreatment with GHRH agonist blunts PLY-induced permeability in control siRNA-treated HL-MVEC, but fails to improve barrier function in PKA-depleted PLY-treated monolayers.

Conclusions: GHRH signaling in HL-MVEC protects from both LPS and PLY-mediated endothelial barrier dysfunction and concurrently induces a barrier-protective PKA-mediated and a barrier-disruptive PKC- $\alpha$-induced pathway in the presence of PLY, the former of which dominates the latter.

Keywords: capillary leak, pneumolysin, lipopolysaccharide, growth hormone-releasing hormone, protein kinase A, protein kinase $\mathrm{C}$

\section{INTRODUCTION}

Of the approximately 9 million children aged less than 5 years that die each year worldwide, 1.6 million succumb from pneumonia (Rudan et al., 2008). Indeed, pneumonia kills more children world-wide than any other single illness. In the US more than 5 million cases of community-acquired pneumonia (CAP) are diagnosed annually, mainly in the elderly population, with $7 \%$ of them fatal (Waterer et al., 2011). Despite the widespread use 
of antibiotics, mortality during the first several days of pneumonia has not decreased appreciably over the past 75 years (Evans and Gaisford, 1938; Waterer et al., 2011). Even though the lungs are essentially free of bacteria following antibiotic treatment, many patients still die from complications, the most important one of which is pulmonary permeability edema. One of the main causes of pneumonia-associated permeability edema in patients treated with antibiotics is the massive release of toxins, including pneumolysin (PLY), a virulence factor from $\mathrm{G}^{+}$pneumococci and lipopolysaccharide (LPS), an endotoxin from $\mathrm{G}^{-}$bacteria (Anderson et al., 2007), following bacterial lysis. Although inducing very different pathways leading to endothelial dysfunction, both toxins are important mediators of pneumonia-associated capillary leak, which is an important problem contributing to permeability edema.

Endothelial and epithelial barrier functions are regulated by a tight balance between opposing centripetal and centrifugal intracellular forces, provided by the contractile machinery and the elements opposing contraction, respectively. The elements opposing barrier dysfunction include tethering complexes, involved in cell-cell and cell-matrix contacts, and systems that impart cell rigidity and prevent cellular collapse, such as actin filaments, microtubules, and intermediate filaments (Dudek and Garcia, 2001; Lucas et al., 2009).

The $53 \mathrm{kDa}$ cytoplasmic cholesterol-binding and poreforming cytolysin, PLY, is a major pneumococcal virulence factor, and exerts its barrier-disruptive effects mainly through its direct activities on myosin light chains (MLC) and on microtubules (Rubins et al., 1996; Witzenrath et al., 2006; Lucas et al., 2012a,b, 2013), rather than through stimulation of leukocyte infiltration and cytokine generation (Maus et al., 2004). By contrast, the $\mathrm{G}^{-}$endotoxin, LPS, upon stimulation of TLR4 (Beutler and Moresco, 2008), directly affects endothelial barrier function, by means of a mechanism involving RhoA GTPase activationdependent downregulation of expression of the adherens junction protein, VE-cadherin (Schlegel et al., 2009; Rafikov et al., 2014). LPS-induced endothelial barrier dysfunction can be blunted by cAMP-dependent activation of protein kinase A (PKA) (Csortos et al., 2008; Bogatcheva et al., 2009; Umapathy et al., 2010). In contrast to PLY, LPS also indirectly promotes hyperpermeability, by means of potently activating leukocyte infiltration, inducing oxidative and nitrosative stress. In addition, increased generation of pro-inflammatory cytokines (Umapathy et al., 2012; Aggarwal et al., 2014; Gonzales et al., 2014), such as TNF, dramatically affects microtubule arrangement in the lung endothelium (Petrache et al., 2003). Currently, no standard treatment exists to counteract LPS- or PLY-mediated permeability edema. As such, the search for novel protective substances which can blunt endothelial barrier dysfunction induced by these bacterial toxins is of high clinical importance.

Generated in the posterior hypothalamus, the nominative function of the Growth Hormone Releasing Hormone (GHRH) is the stimulation of Growth Hormone production by the pituitary, which expresses full length GHRH receptors (GHRH-R) (Mayo et al., 1995; Sherwood et al., 2000). We have previously shown that mRNA for the ligand GHRH and its bioactive receptor splice variant, SV1, are also expressed in peripheral tissues, such as in lung microvascular endothelial cells, pancreatic islets, and cardiomyocytes (Rekasi et al., 2000; Kanashiro-Takeuchi et al., 2010; Kiaris et al., 2011; Ludwig et al., 2012).

The $29 \mathrm{~N}$-terminal amino acid residues of the GHRH sequence possess full biological activity and thus constitute the core peptide for the development of agonists of GHRH, such as JI-34, JI-36, and JI-38, which are up to 80 times more potent than GHRH (Izdebski et al., 1995; Cai et al., 2014). JI-34 was shown to protect from PLY-induced barrier dysfunction in vitro and in vivo, in a cAMP-dependent manner (Lucas et al., 2012a). However, the outcome of GHRH agonist treatment on LPS-induced hyperpermeability in capillary endothelial cells in vitro and in vivo has not been investigated.

Ligand or agonist binding to the GHRH-R changes receptor conformation and activates the closely associated heterotrimeric Gs-protein. Upon this activation, the dissociated Gas subunit directly stimulates adenylate cyclase and intracellular cAMP generation, which in turn activates PKA (Moretti et al., 2002). However, GHRH belongs to the glucagon/secretin superfamily that has been demonstrated to activate receptors coupled with multiple heterotrimeric $G$ proteins, particularly Gs and Gq (Moretti et al., 2002; Lania et al., 2003). Therefore, in addition to cAMP accumulation and PKA activation via Gs, these receptors may also potentially induce a rise in intracellular $\mathrm{Ca}^{2+}$ and PKC activation via Gq (Lania et al., 2003).

The main aims of this study were to investigate whether apart from PLY, GHRH agonists can also protect from LPS-induced barrier dysfunction. We also investigated whether GHRH agonists are able to activate both PKA-mediated barrier-protective and PKC-mediated barrier-disruptive pathways in human lung microvascular endothelial cells (HL-MVEC) and how these pathways interact.

\section{MATERIALS AND METHODS CELLS}

Human lung microvascular endothelial cells (HL-MVEC) and human pulmonary artery endothelial cells (HPAEC) (Lonza, Walkersville, MD, USA) were grown in complete EBM-2 medium (Lonza, Walkersville, MD, USA) and used up to passage six. Experiments with PLY were performed in serum-free medium, whereas experiments with LPS were performed in medium containing 5\% FBS.

\section{MICE}

Eight to ten weeks old male C57BL6 mice, weighing 19-21 g were obtained from Harlan and were kept at the animal facilities at Georgia Regents University. All animal studies conformed to National Institutes of Health guidelines. The experimental procedure was approved by the Georgia Regents University Institutional Animal Care and Use Committee.

\section{PLY PURIFICATION}

PLY was purified from a recombinant Listeria innocua 6a strain expressing LPS-free PLY in the laboratory of T.C. The batch of PLY used in this study had a specific activity of $1.25 \times 10^{7}$ hemolytic units/mg. 


\section{BIOCHEMICALS}

Rabbit polyclonal anti-human vascular endothelial (VE)-cadherin antibodies, anti-human PKA, anti-human phospho-PKA, anti-human PKA substrate, anti-human PKC substrate, and anti-human PKC- $\alpha$ were from Cell Signaling Technology (Danvers, MA, USA). Rabbit anti-human phospho PKC- $\alpha$ (Ser 657) was from Santa Cruz Biotechnology (Dallas, TX, USA). Secondary goat anti-rabbit Texas Red-conjugated antibody and goat anti-rabbit secondary antibodies conjugated to Horse Radish Peroxidase (HRP) were from Sigma-Aldrich (St Louis, MO). LPS E. coli 0111:B4 was from Sigma (St Louis, MO).

\section{PEPTIDE ANALOGS PREPARATION}

GHRH agonist, JI-34, was synthesized in the laboratory of A.V.S. and is 80 times more potent in stimulating the GHRH-R than GHRH (Izdebski et al., 1995). For preparation of the stock solution, the agonist was dissolved in DMSO.

\section{DEPLETION OF PKA $\alpha$ CATALYTIC SUBUNIT AND PKC- $\alpha$ IN HL-MVEC}

HL-MVEC were treated with a pool of 3 target-specific 19-25 nt siRNAs designed to knock down either PKA $\alpha$ catalytic subunit or PKC- $\alpha$ gene expression. These were obtained from Santa Cruz Biotechnology, Inc. (Dallas, TX, USA). Also a non-specific, non-targeting siRNA was ordered from the same manufacturer. All siRNA's were received in lyophilized form. HL-MVEC were transfected at $70-80 \%$ confluence with $75 \mathrm{nM}$ final concentration of siRNA using siPORT ${ }^{\mathrm{TM}}$ Amine transfection reagent (Ambion, Life Technologies, Grand Island, NY) and used for further experiments at $48 \mathrm{~h}$ post transfection.

\section{WESTERN BLOTTING PROCEDURE}

Immediately after treatment, HL-MVEC were washed twice with ice-cold PBS and lysed with lysis buffer [20 mM Tris. $\mathrm{HCl}$ ( $\mathrm{pH}$ 7.6), $0.5 \%$ Nonidet P- $40,250 \mathrm{mM} \mathrm{NaCl}, 3 \mathrm{mM}$ EDTA, $3 \mathrm{mM}$ EGTA, $1 \mathrm{mM}$ DTT, and protease inhibitor mixture]. The clear supernatants after centrifugation were mixed with SDS sample buffer and boiled for $5 \mathrm{~min}$. Protein extracts were separated on SDS/PAGE, transferred to a nitrocellulose membrane, incubated with primary antibodies, and then incubated with HRPconjugated secondary antibody. The immunoreactive proteins were visualized with LumiGLO solution (Cell Signaling, Danvers, MA, USA) and were then exposed to X-ray film. The relative intensity of each protein band was quantified with ImageJ software (National Institutes of Health).

\section{MEASUREMENTS OF VE-CADHERIN EXPRESSION ZONE WIDTH}

Analysis of VE cadherin zone expression was performed on immunofluorescent images we prepared using primary rabbit polyclonal anti-human VE-cadherin antibodies (Cell signaling, Danvers, MA, USA) and secondary goat anti-rabbit Texas Redconjugated antibody (Sigma-Aldrich, St Louis, MO, USA) in HPAECs, which are better suited for this type of measurement than pulmonary microvascular endothelial cells. Images were acquired using a N-SIM super-resolution system, assembled on a TiE inverted microscope (Nikon, Japan) with a $100 \times$ oil immersion lens $(N A=1.49)$ and an iXon-897 EMCCD-camera (Andor, Ireland, effective pixel size $60 \mathrm{~nm}$ ). Original images were processed using ImageJ software (Gaussian filtration and background subtraction). Measurements of the width of VE-cadherin expression zone were produced using ImageJ software (function "Measure"). Statistical analysis was performed using Sigma Plot 7.1 (SPSS Science, Point Richmond, CA) and Excel. Sigma Plot 7.1 software was used for graphical data presentation.

\section{MEASUREMENT OF TRANSENDOTHELIAL ELECTRICAL RESISTANCE (TER)}

TER in HL-MVEC monolayers [electrical cell-substrate impedance sensing (ECIS) system 1600R; Applied Biophysics, Troy, NY, USA] was measured as described previously (Lucas et al., 2012a,b).

\section{ASSESSMENT OF CAPILLARY LEAK IN VIVO}

Mice ( $n=10$ per group), pretreated i.v. or not at $-24 \mathrm{~h}$ and at $-1 \mathrm{~h}$ before toxin instillation with JI-34 $(100 \mu \mathrm{g} / \mathrm{kg})$ were anesthetized with i.p. ketamine $(150 \mathrm{mg} / \mathrm{kg})$ and acetylpromazine $(15 \mathrm{mg} / \mathrm{kg})$. Subsequently the trachea was exposed and LPS (Sigma-Aldrich, E. coli 0111:B4, $0.65 \mathrm{mg} / \mathrm{kg}$ in saline) was instilled i.t. for $24 \mathrm{~h}$ in anesthetized mice via a 20-gauge catheter. An Evans Blue dye (EBD)/Albumin mixture $(30 \mathrm{mg} / \mathrm{kg}$ in saline; $(0.5 \%$ EBD conjugated to $4 \%$ BSA, Fraction V; Sigma-Aldrich, St Louis, $\mathrm{MO}$ ) was injected into the tail vein of 6 mice per group, $2 \mathrm{~h}$ before mice were killed, in order to assess vascular leak. Lungs free of blood were weighed and snap-frozen in liquid nitrogen. The left lung was homogenized, incubated with formamide $(18 \mathrm{~h}$ at $+60^{\circ} \mathrm{C}$ ), and centrifuged at $5000 \times \mathrm{g}$ for $30 \mathrm{~min}$. The optical density of the supernatant was determined spectrophotometrically at $620-750 \mathrm{~nm}$. Extravasated EBD concentration in the lungs was calculated by using a standard curve ( $\mu \mathrm{g}$ of EBD per $\mathrm{g}$ of wet lung tissue), as described previously (Lucas et al., 2012a,b). For pro-inflammatory mediator determination, collected bronchoalveolar lavage fluid (BALF) from another 4 mice per group was centrifuged $\left(500 \times \mathrm{g}\right.$ for $15 \mathrm{~min}$ at $\left.4^{\circ} \mathrm{C}\right)$, supernatant was centrifuged again $\left(5000 \times \mathrm{g}\right.$ for $15 \mathrm{~min}$ at $\left.4^{\circ} \mathrm{C}\right)$, and pure BALF was used to measure cytokine/chemokine/growth factor concentrations using the multiplex MCYTOMAG-70K assay (EMD Millipore, Billerica, MA, USA), according to the manufacturer's instructions.

\section{STATISTICAL ANALYSIS}

All experimental data are presented as mean \pm SD. Control samples and those obtained upon various stimuli were compared by unpaired Student's $t$-test. For multiple group comparisons, One-Way ANOVA was used. $p<0.05$ was considered statistically significant.

\section{RESULTS \\ GHRH AGONIST PRESERVES VE-CADHERIN EXPRESSION IN LPS-TREATED HUMAN ENDOTHELIAL MONOLAYERS IN VITRO}

The main function of VE-cadherin, a crucial component of endothelial adherens junctions, is the stabilization of endothelial cell integrity. VE-cadherin expression was shown to be significantly reduced in the endothelium of all vessel types in the lungs of $\mathrm{G}^{-}$-sepsis-induced acute respiratory distress syndrome (ARDS) or in endothelial cells treated with LPS 
(Herwig et al., 2013). As shown in Figures 1A,B, treatment of monolayers of HPAECs for $6 \mathrm{~h}$ with LPS $(500 \mathrm{ng} / \mathrm{ml})$ leads to a significant reduction in VE-cadherin expression, measured as the mean of the VE-cadherin zone width (in $\mu \mathrm{m}$ ). Pretreatment of the monolayers for $15 \mathrm{~min}$ with the GHRH agonist, JI-34 $(1 \mu \mathrm{M})$, before LPS addition significantly blunted the effect of LPS and increased VE-cadherin expression even in control cells. In view of the crucial role of VE-cadherin expression for barrier function, these results indicate a barrier-protective effect of GHRH in LPS-treated endothelial cells in vitro. Since LPS exerts both direct and indirect (cytokine-mediated) deleterious actions on barrier function in the lung in vivo, in the subsequent study we investigated whether JI-34 can also blunt LPS-induced capillary leak in mice.

\section{JI-34 BLUNTS LPS-INDUCED PULMONARY CAPILLARY LEAK IN VIVO}

As shown in Figure 2, a combined i.v. pretreatment of 8 weeks old male C57BL6 mice $(n=6)$, with JI-34 $(100 \mu \mathrm{g} / \mathrm{kg})$ at 24 and $1 \mathrm{~h}$ prior to i.t. LPS instillation $(0.65 \mathrm{mg} / \mathrm{kg})$ induced a significant protection from LPS-induced capillary leak, as compared to vehicle control and measured as EBD incorporation in the lung tissue. As depicted in Figure 3, JI-34 did not affect the concentration of the pro-inflammatory cytokines TNF, IL-1 $\beta$, and IL- 6 or of the anti-inflammatory cytokine IL-10 in BALF of LPS-treated animals, as measured by multiplex analysis.

Moreover, neither the generation of the pro-inflammatory leukocyte-attracting chemokines MCP-1, MIP-1 $\beta$, and MIP-2 nor of the permeability-increasing factor, VEGF, were affected by the GHRH agonist in LPS-instilled mice (Figure 4). These results suggest that the barrier-protective effect of JI-34 in lungs of LPStreated mice results mainly from blunting direct, rather than indirect, leukocyte-mediated effects of LPS.

\section{CRUCIAL ROLE FOR PROTEIN KINASE A IN BARRIER-PROTECTIVE ACTIVITY OF GHRH AGONISTS IN HUMAN LUNG MICROVASCULAR ENDOTHELIAL CELLS}

In endothelial cells, cAMP potentiates VE-cadherin-mediated cell-cell contacts, causing enhanced endothelial barrier function (Dejana et al., 2008). The observed inhibitory activity of the GHRH agonist, JI-34, on both PLY-mediated (Lucas et al., 2012a) and LPS-induced VE-cadherin loss (Figures 1A,B), can thus, at least partially, be explained by its capacity to activate cAMP, upon activating the SV1 receptor, expressed in HL-MVEC. cAMP can however activate both PKA and Epac. PKA exerts its barrier-protective effects upon phosphorylating its substrates, including VASP, which was shown to be important in protecting endothelial barriers from LPS action (Bogatcheva et al., 2009). By contrast, Epac activates the barrier protective Rap1/Rac 1 cascade (Fukuhara et al., 2005).

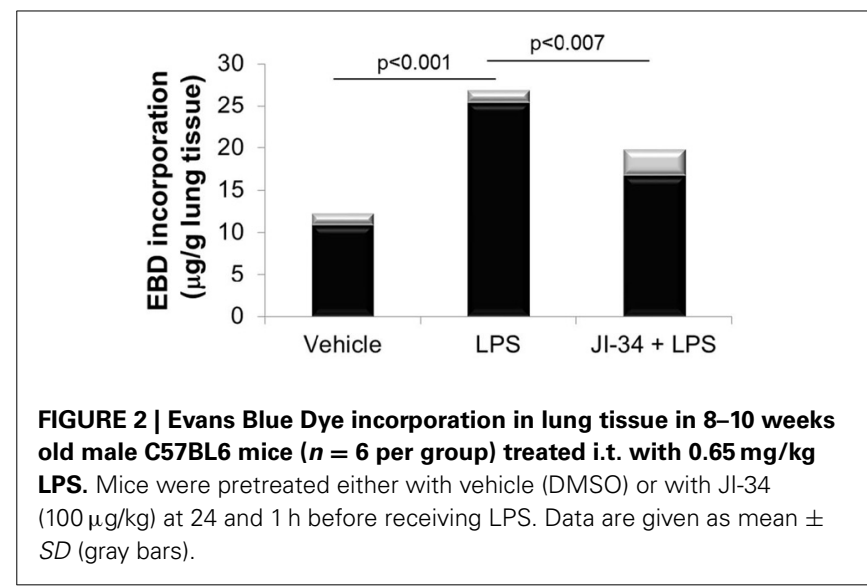

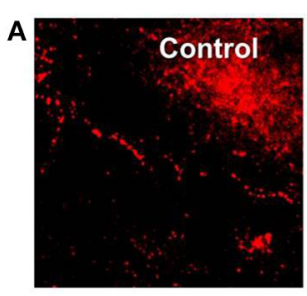
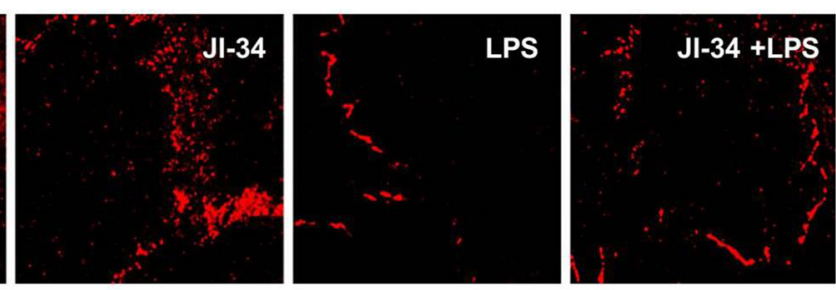

B

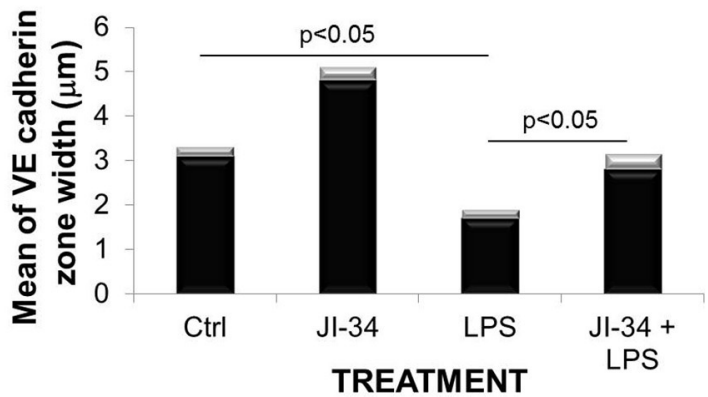

FIGURE 1 | Effect of LPS and JI-34 on VE-cadherin adherens junction of human pulmonary artery endothelial cells (HPAEC). (A) Cells were pretreated with JI-34 (1 $\mu \mathrm{M}, 15 \mathrm{~min}$ ) or not, followed by LPS stimulation (500 ng/ml, $6 \mathrm{~h}$ ) and stained with VE-cadherin antibodies for VE-cadherin adherens junction protein detection. (B) Measurements of VE-cadherin expression zone width were carried out on immunofluorescent images using ImageJ software. Mean of VE-cadherin zone width are presented as $\pm S D$ (gray bars) ( $n=100$ for each experimental treatment). 
A

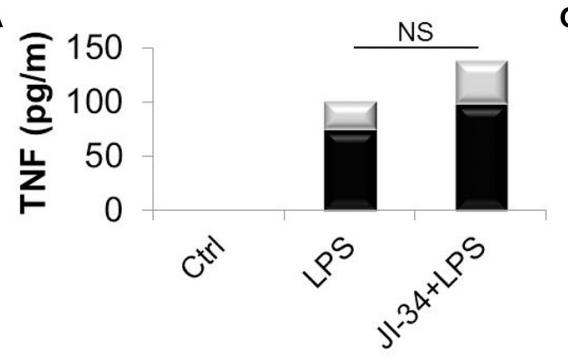

TREATMENT

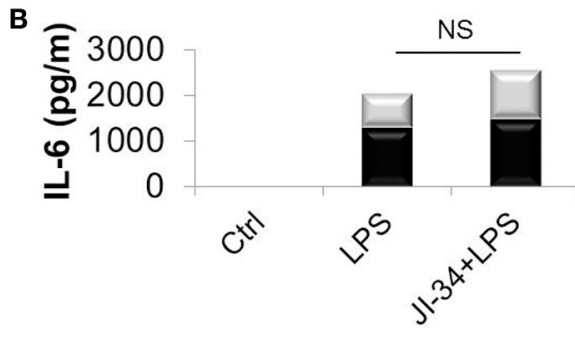

TREATMENT
FIGURE 3 | Concentrations of (A) TNF; (B) IL-6; (C) IL-1 $\beta$; and (D) IL-10 in BALF from vehicle-treated, LPS-treated $(0.65 \mathrm{mg} / \mathrm{kg})$ or $\mathrm{Jl}-34(100 \mu \mathrm{g} / \mathrm{kg})+$ LPS treated mice $(n=4$ per group) in $\mathrm{pg} / \mathrm{ml}$, as assessed using the Multiplex MCYTOMAG-70K assay (EMD Millipore, Billerica, MA, USA). Data are presented as mean $\pm S D$ (gray bars).
A

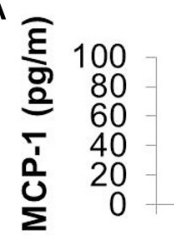

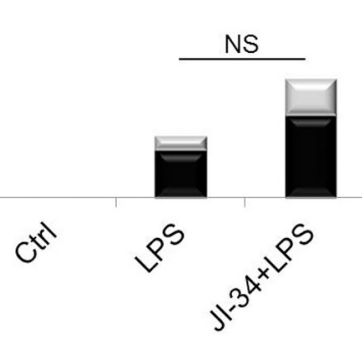

TREATMENT

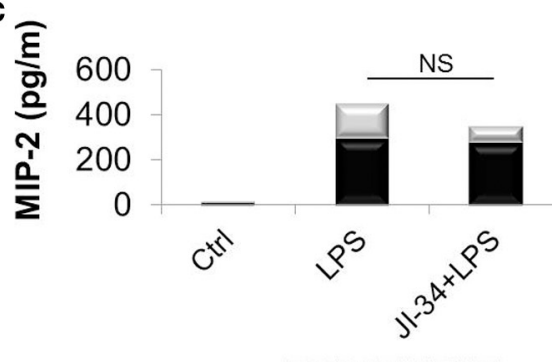

TREATMENT

FIGURE 4 | Concentrations of the chemokines (A) MCP-1; (B) MIP-1 $\beta$; (C) MIP-2; and the growth factor (D). VEGF in BALF from

vehicle-treated, LPS-treated or JI-34 + LPS treated mice $(n=4$ per

Since agonists of both PKA and Epac can independently protect from LPS-induced hyperpermeability (Bogatcheva et al., 2009), we investigated whether specific activation of Epac, using the agonist 2'-O-Methyl-cAMP, can protect from PLY-mediated

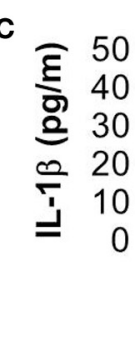

D

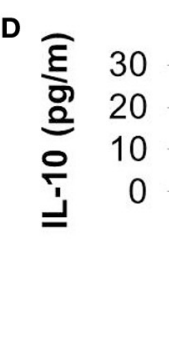

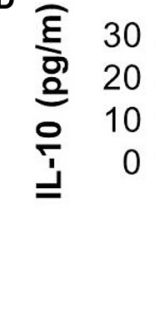
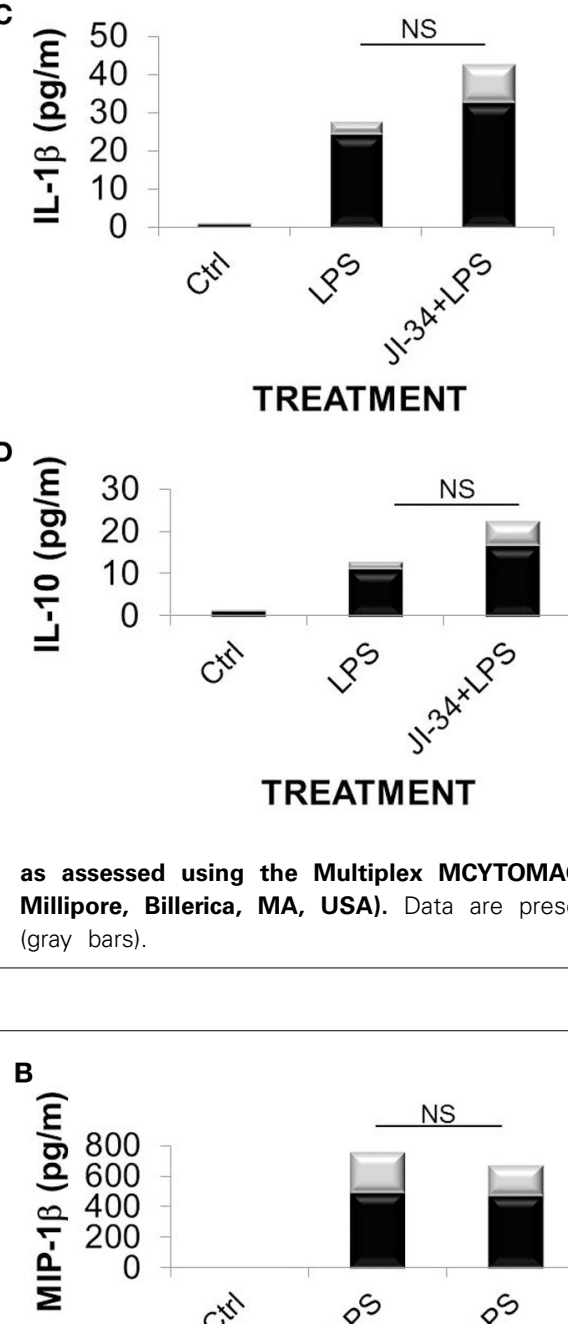

TREATMENT

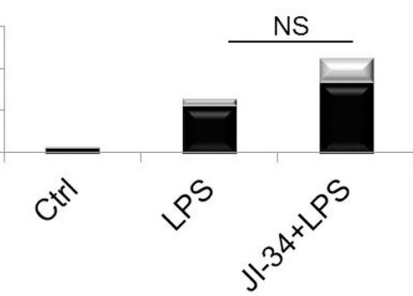

TREATMENT 


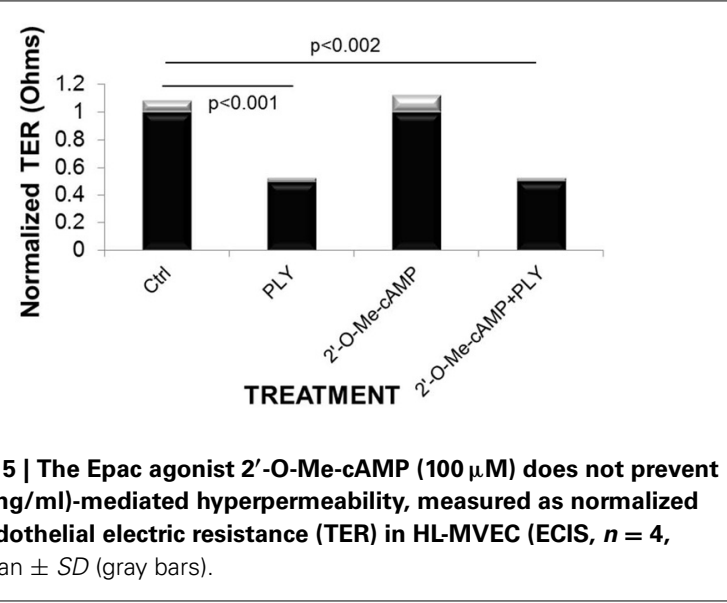

(Bogatcheva et al., 2009). Since we have previously shown that cAMP protects from PLY-mediated barrier dysfunction in monolayers of HL-MVEC (Umapathy et al., 2010), this result suggests that the protective effect of the GHRH agonist JI-34 in PLYmediated barrier dysfunction is dependent on the activation of PKA, rather than of Epac.

In order to substantiate this hypothesis, we investigated whether JI-34 can activate PKA in the absence or presence of LPS or PLY. As shown in Figure 6A, JI-34 (1 $\mu \mathrm{M})$ increases PKA activity, assessed as the expression of PKA-phosphorylated substrate protein in Western blotting in both resting and LPS-treated HL-MVEC monolayers. Further, as shown in Figures 6B,C, JI-34 $(1 \mu \mathrm{M})$ also activates PKA in PLY (30 ng/ml)-treated HL-MVEC, measured as the increased expression of phosphorylated over total PKA. Taken together, these results indicate that the activation of PKA, rather than of Epac, mediates the protective activity of JI-34 in both LPS- and PLY-mediated barrier dysfunction.

\section{ACTIVATION OF SV-1 RECEPTOR IN HL-MVEC CAN STIMULATE BACTERIAL TOXIN-INDUCED ACTIVATION OF PKC- $\alpha$}

GHRH is a member of the glucagon/secretin superfamily, the members of which have the capacity to activate receptors coupled with multiple heterotrimeric Gq proteins, which have the capacity to increase intracellular $\mathrm{Ca}^{2+}$ (Mayo et al., 1995; Sherwood et al., 2000). The Gaq subunit, which was reported to be activated upon GHRH-R stimulation, has the capacity to activate PKC, after first stimulating phospholipase C (PLC) (Lania et al., 2003). In this study, we therefore investigated whether a $30 \mathrm{~min}$ incubation with JI-34 $(1 \mu \mathrm{M})$ of both resting cells and PLY-treated cells can increase PKC activity, assessed as the expression of PKCphosphorylated protein substrate in Western Blotting. As shown in Figure 7A, a 30 min pretreatment of HL-MVEC monolayers with JI-34 $(1 \mu \mathrm{M})$ increases the expression of protein substrates phosphorylated by PKC in the presence of PLY $(15 \mathrm{ng} / \mathrm{ml})$, which by itself activates this process within $15 \mathrm{~min}$. One of the most important PKC isoforms which is activated in JI-34/PLY-treated cells is PKC- $\alpha$ (Figure 7B, upper panel), since siRNA-mediated PKC- $\alpha$ gene depletion significantly reduces JI-34/PLY-mediated phosphorylation of PKC substrates in these cells (Figure 7B, lower panel). Interestingly, as shown in Figures 8A,B, JI-34 did

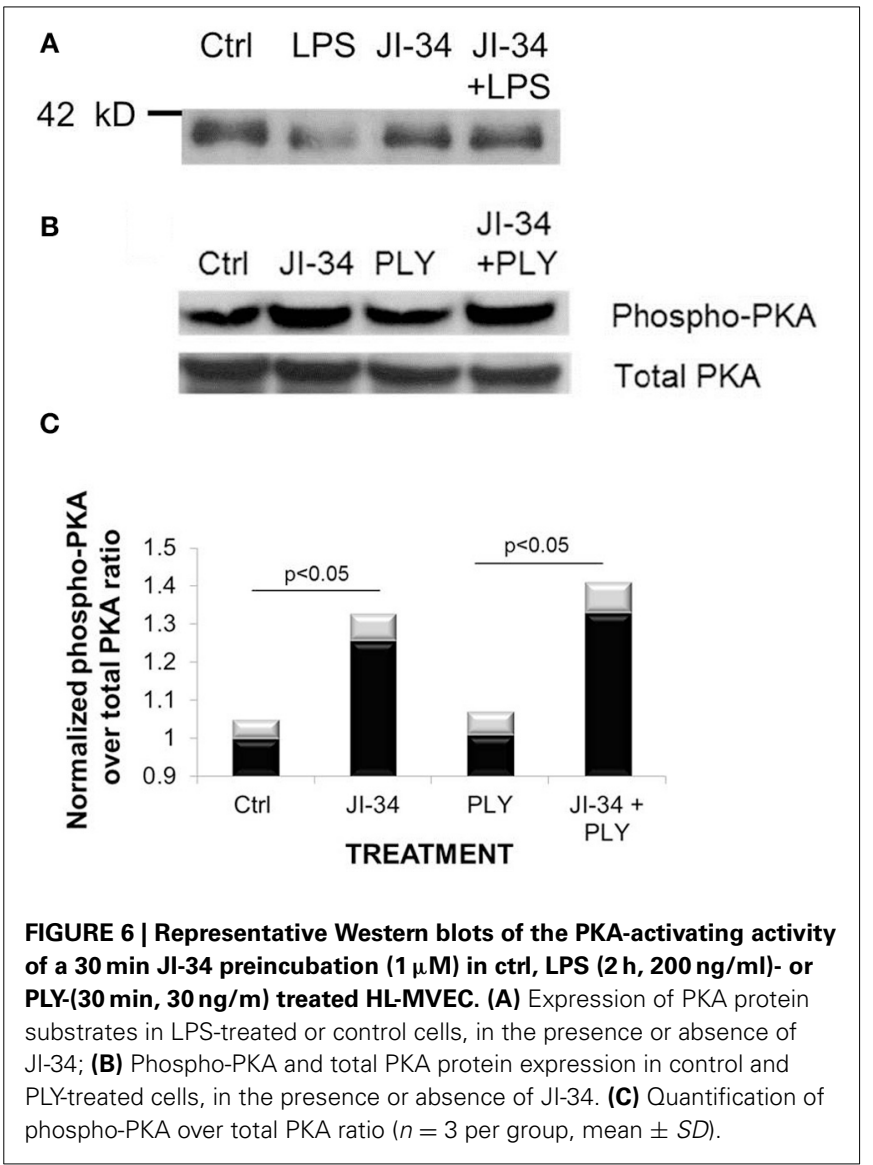

not further increase LPS $(1 \mu \mathrm{g} / \mathrm{ml})$-induced PKC- $\alpha$ activation, in contrast to what we observed in PLY-treated cells.

\section{PKA GENE DEPLETION BLUNTS THE BARRIER-PROTECTIVE ACTIVITY OF GHRH AGONIST IN PLY-TREATED HL-MVEC MONOLAYERS}

In view of our previous observations that JI-34 treatment can increase both barrier-protective PKA activity and barrierdisruptive PKC- $\alpha$ signaling in the presence of PLY, we next investigated the outcome of siRNA-mediated gene depletion of the PKA $\alpha$ catalytic subunit, on the JI-34-induced PKC activation in HL-MVEC monolayers. As shown in Figure 9, PKA $\alpha$ catalytic site (cat) gene depletion significantly increases basal and JI-34induced PKC activity, measured as the expression of phosphorylated PKC protein substrates, as compared to scrambled siRNA transfection.

As shown in Figure 10A, PKA gene depletion blunts the protective effect of JI-34 toward PLY $(30 \mathrm{ng} / \mathrm{ml})$, seen in scrambled siRNA-treated cells. Monolayer resistance of untreated scrambled siRNA-treated HL-MVEC monolayers is not significantly different from untreated control cells (data not shown). PKA $\alpha$ cat siRNA-transfected cells display an increased sensitivity to PLY (Figures 10A,B). The latter result is somewhat in contrast with the equal total PKC activity detected in Western blotting (Figure 9), but could represent an increased activation of barrierdisruptive (such as PKC- $\alpha$ ) over barrier-protective PKC isoforms (such as PKC- $\delta$ ) in HL-MVEC, which would not necessarily be 


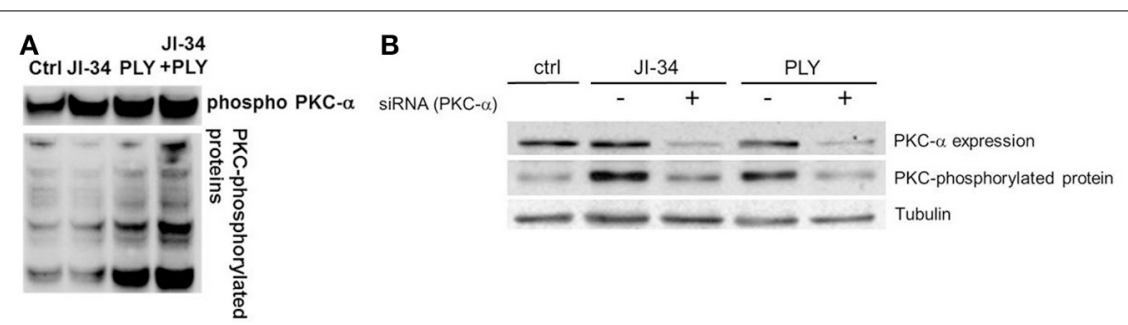

FIGURE 7 | (A) Expression of PKC-phosphorylated protein substrates in ctrl, PLY (30 ng/ml), Jl-34 (1 $\mu \mathrm{M})$, and Jl-34 + PLY-treated HL-MVEC. Cells were pretreated for $30 \mathrm{~min}$ with $\mathrm{Jl}-34$ and were then incubated for
15 min with PLY. (B) Representative WB of the Influence of siRNA-mediated PKC- $\alpha$ gene depletion on PLY- and JI-34 induced total PKC activity in HL-MVEC.

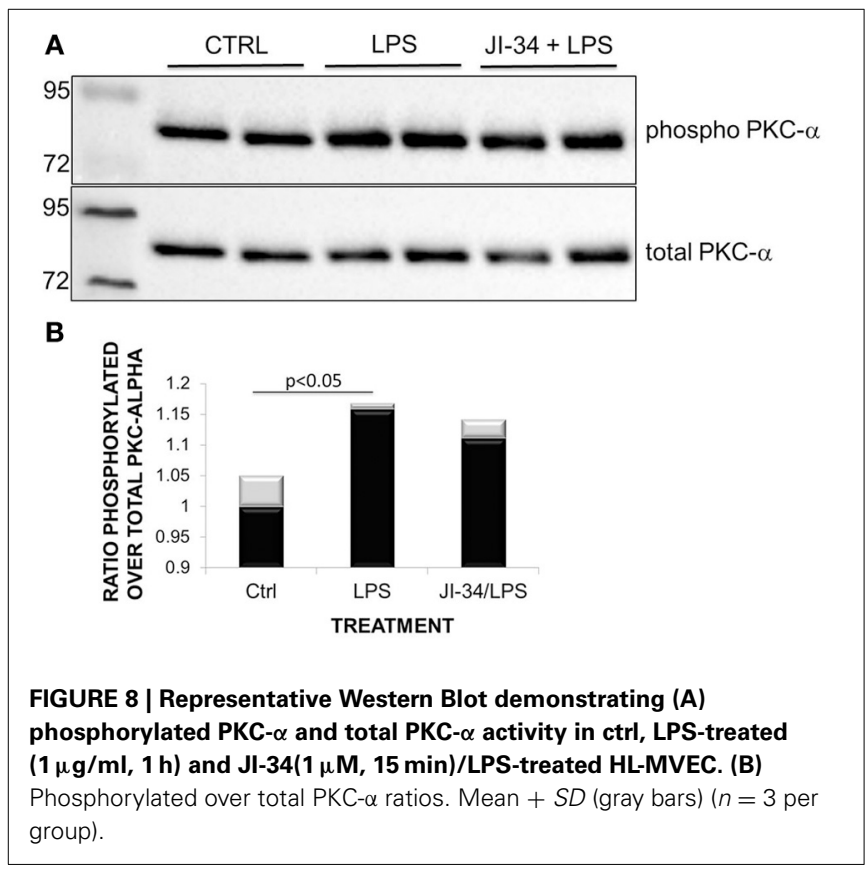

SiRNA (PKA)
PKC-phosphorylated
proteins

detected in the total PKC activity. As shown in Figure 10B, at a lower PLY concentration $(15 \mathrm{ng} / \mathrm{ml})$, the protective effect of JI-34 may shift even to a deleterious one in PKA-gene depleted cells.

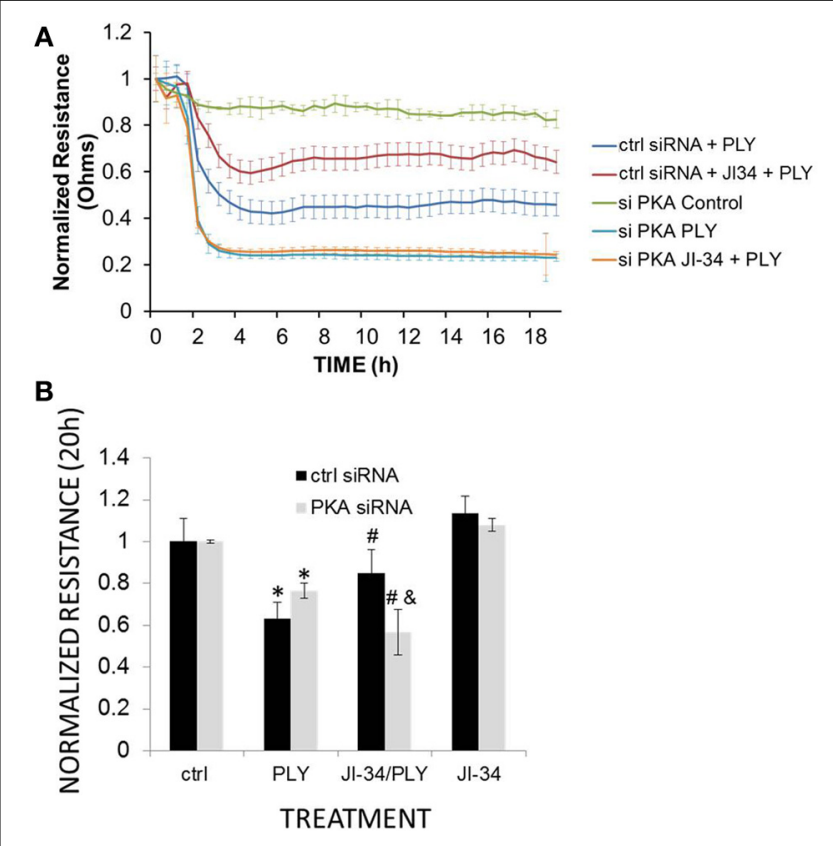

FIGURE 10 | (A) TER of HL-MVEC monolayers transfected with either scrambled (control) or PKA $\alpha$ cat. siRNA, that were treated with vehicle, JI-34 (1 $\mu \mathrm{M})$, PLY (30 ng/ml), or JI-34 + PLY. N = 4 per group and data are depicted as mean $\pm S D$. (B) TER at $20 \mathrm{~h}$ of ctrl or PKA-depleted HLMVEC treated with JI-34 (1 $\mu \mathrm{M})$, PLY (15 ng/ml), or Jl-34 + PLY. * $p<0.05$ vs. ctrl; ${ }^{\#} p<0.05$ vs. PLY in control siRNA-treated cells; $\#, \&<0.05$ vs. PLY in siRNA (PKA) transfected cells.

\section{DISCUSSION}

Both the GHRH-R and the bioactive SV1 receptor, the latter of which is expressed in HL-MVEC, are coupled to heterotrimeric G proteins (Mayo et al., 1995; Sherwood et al., 2000; Kiaris et al., 2011). The latter consist of $\alpha, \beta$, and $\gamma$ subunits and function as transducers of signals from G-protein coupled receptors (GPCRs). Whereas the $\alpha$ subunit is the GTP binding protein, the $\beta$ and $\gamma$ subunits are anchored to the plasma membrane and bind the GDP-bound $\alpha$ subunit with high affinity to constitute the heterotrimer. Although the barrier-protective roles of cAMP and of PKA are well-documented (Bogatcheva et al., 2009), the JI-34-induced interplay between the barrier-protective $\mathrm{PKA}$, induced upon $\mathrm{G} \alpha \mathrm{S}$ activation and the barrier-disruptive PKC- $\alpha$, activated by G $\alpha \mathrm{q}$, represents a unique situation. This 
can be especially relevant in the presence of bacterial toxins, such as PLY and LPS, which were both shown to activate PKC- $\alpha$ (Witzenrath et al., 2006; Lucas et al., 2012b; Zhao et al., 2013). Thus, unraveling the mechanism by which agonists of GHRH exert their protective effect under these complex circumstances is important. Pathways promoting hyperpermeability in PLY- or LPS-treated MVEC include both MLC-dependent mechanisms and microtubule de-polymerization, the latter of which can cause disassembly of adherens junction proteins, such as VE-cadherin (Petrache et al., 2003; Lucas et al., 2012a). By contrast, activation of PKA, by means of CAMP, was shown to stabilize barrier function in the presence of LPS and PLY (Bogatcheva et al., 2009; Umapathy et al., 2010).

We show in this study that the GHRH agonist JI-34 not only protects HL-MVEC monolayers from PLY-induced barrier dysfunction, but also from LPS-mediated impairment of VE cadherin expression. This adherens junction protein is a relevant parameter of barrier function during acute lung injury. Indeed, a significant reduction of VE cadherin expression was recently detected in lung capillaries of patients with $\mathrm{G}^{-}$sepsis-induced ARDS (Herwig et al., 2013). As such, the VE cadherin expressionrestoring activity of JI-34 can provide a plausible explanation for the observed protection from capillary leak in mice which have been instilled i.t. with LPS. Indeed, the improved barrier function in GHRH agonist/LPS-treated animals is not a consequence of a reduced inflammatory response, but rather of an inhibition of direct effects of LPS on the integrity of the lung capillaries. Our data have also shown that of the two pathways induced by cAMP generation following G $\alpha$ S activation, Epac and PKA, in particular the latter seems to be crucial for the protective effect of the GHRH agonist in PLY-treated cells. Indeed, the Epac agonist 2'-O-Me-cAMP failed to protect HL-MVEC monolayers from PLY-mediated barrier dysfunction, in contrast to CAMP (Umapathy et al., 2010) and siRNA-mediated depletion of PKA completely blunted the protective effect of JI-34.

Activation of PKA has been demonstrated to attenuate both epithelial and endothelial barrier dysfunction (Lawrence et al., 2002; Fukuhara et al., 2005). In the endothelium, PKA activation can blunt ROCK activity by direct phosphorylation of RhoGDI and prevention of the release of RhoA from the RhoA-RhoGDI complex (Qiao et al., 2008). Cyclic AMP potentiates VE-cadherinmediated cell-cell contacts, causing enhanced endothelial barrier function (Dejana et al., 2008). Hence, our observed inhibitory activity of GHRH agonist, JI-34, on PLY- and LPS-mediated VEcadherin loss can at least partially be explained by its capacity to activate cAMP/PKA pathway, upon binding to the SV1 receptor, expressed in HL-MVEC.

As demonstrated in this study, JI-34 treatment, beyond activating PKA in PLY-treated HL-MVEC, also further increases PLY-induced activation of the PKC- $\alpha$ isoform of PKC. This kinase mediates PLY-induced endothelial hyperpermeability, upon stimulating the barrier disruptive RhoA/ROCK pathway, which increases MLC phosphorylation, actomyosin contractility and intercellular gap formation. Although JI-34 increases PLYinduced PKC- $\alpha$ activity, it does not affect LPS-induced activation of this enzyme. This could potentially be linked to the fact that LPS increases several PKC isoforms, whereas PLY, which induces a large influx of extracellular $\mathrm{Ca}^{2+}$ upon pore formation in $\mathrm{HL}-$ MVEC, can preferentially activate $\mathrm{Ca}^{2+}$-dependent isoforms, of which PKC- $\alpha$ is the main representative.

Although the stimulation of the SV1 receptor in HL-MVEC induces two apparently opposing pathways in the presence of PLY. On the one hand, GHRH agonist stimulates the barrierprotective CAMP/PKA pathway and on the other hand it increases the barrier-disruptive PKC- $\alpha /$ RhoA/ROCK pathway. Our results demonstrate an overriding role for the PKA pathway over the PKC- $\alpha$ pathway under these conditions. However, as shown in Figures 10A,B, when the PKA pathway is impaired, the PKC pathway prevails. Taken together, these results suggest that GHRH agonists will protect from PLY-induced hyperpermeability provided the CAMP/PKA pathway is intact.

Lesions of specific anatomical sites in the brainstem and hypothalamus may initiate acute pulmonary edema. This occurrence has been termed neurogenic pulmonary edema. Unfortunately, the pathophysiologic explanation of this response is not clearly understood. Although hydrostatic derangements may explain certain cases of neurogenic pulmonary edema, recent clinical and experimental studies have indicated that central nervous system disorders may cause a permeability defect without a vascular insult (Colice et al., 1984). The mediating factor for this permeability defect is not clear. It is therefore tempting to hypothesize that peripheral actions of GHRH produced in the hypothalamus may control pulmonary barrier integrity. When this production is impaired, as may occur from brainstem lesions, this may affect pulmonary barrier integrity.

Since GHRH agonists have a longer half-life than the native hypothalamic GHRH hormone (Izdebski et al., 1995; Cai et al., 2014), they can provide a more potent signal to damaged lungs and could promote a change in the concept of treating pulmonary edema. GHRH agonists could improve lung function in pathologies associated with pulmonary permeability edema, such as CNS trauma, ARDS, and severe pneumonia.

\section{ACKNOWLEDGMENTS}

Supported by National Institutes of Health/National Heart, Lung and Blood Institute grants R01HL094609 (Rudolf Lucas), RO1HL51854 (Michael A. Matthay), and P01HL101902 (Alexander D. Verin), by Russian Federation for Basic Research grants 09-04-00363 and 12-04-00488 (Irina B. Alieva) and MSU Development Program (5.13), and by a grant from the Deutsche Forschungsgemeinschaft through the Transregio Initiative TRR84 Project A4 (Helena Pillich and Trinad Chakraborty). The Andrew V. Schally laboratory was supported by the Medical Research Service of the Veterans Affairs Department, by the Departments of Pathology and Medicine (Division of Hematology/Oncology, Sylvester Comprehensive Cancer Center) of the Miller School of Medicine at the University of Miami, and by the South Florida Veterans Affairs Foundation for Research and Education. Norman L. Block was supported by the L. Austin Weeks family endowment for Urologic Research.

\section{REFERENCES}

Aggarwal, S., Gross, C. M., Kumar, S., Dimitropoulou, C., Sharma, S., Gorshkov, B. A., et al. (2014). Dimethylarginine dimethylaminohydrolase II overexpression 
attenuates LPS-mediated lung leak in acute lung injury. Am. J. Respir. Cell Mol. Biol. 50, 614-625. doi: 10.1165/rcmb.2013-0193OC

Anderson, R., Steel, H. C., Cockeran, R., Von Gottberg, A., de Gouveia, L., Klugman, K. P., et al. (2007). Comparison of the effects of macrolides, amoxicillin, ceftriaxone, doxycycline, tobramycin and fluoroquinolones, on the production of pneumolysin by Streptococcus pneumoniae in vitro. J. Antimicrob. Chemother. 60, 1155-1158. doi: 10.1093/jac/dkm338

Beutler, B., and Moresco, E. M. (2008). The forward genetic dissection of afferent innate immunity. Curr. Topics Microbiol. Immunol. 321, 3-26.

Bogatcheva, N. V., Zemskova, M. A., Kovalenkov, Y., Poirier, C., and Verin, A. D. (2009). Molecular mechanisms mediating protective effect of cAMP on lipopolysaccharide (LPS)-induced human lung microvascular endothelial cells (HLMVEC) hyperpermeability. J. Cell. Physiol. 221, 750-759. doi: $10.1002 /$ jcp. 21913

Cai, R., Schally, A. V., Cui, T., Szalontay, L., Halmos, G., Sha, W., et al. (2014). Synthesis of new potent agonistic analogs of growth hormone-releasing hormone (GHRH) and evaluation of their endocrine and cardiac activities. Peptides 52, 104-112. doi: 10.1016/j.peptides.2013.12.010

Colice, G. L., Matthay, M. A., Bass, E., and Matthay, R. A. (1984). Neurogenic pulmonary edema. Am. Rev. Respir. Dis. 130, 941-948.

Csortos, C., Czikora, I., Bogatcheva, N. V., Adyshev, D. M., Poirier, C., Olah, G., et al. (2008). TIMAP is a positive regulator of pulmonary endothelial barrier function. Am. J. Physiol. Lung Cell. Mol. Physiol. 295, L440-L450. doi: 10.1152/ajplung.00325.2007

Dejana, E., Orsenigo, F., and Lampugnani, M. G. (2008). The role of adherens junctions and VE-cadherin in the control of vascular permeability. J. Cell Sci. 121(Pt 13), 2115-2122. doi: 10.1242/jcs.017897

Dudek, S. M., and Garcia, J. G. (2001). Cytoskeletal regulation of pulmonary vascular permeability. J. Appl. Physiol. 91, 1487-1500.

Evans, G. M., and Gaisford, W. F. (1938). Treatment of pneumonia with 2-(pAminobenzenesulphonamido) pyridine. Lancet 2, 14-18. doi: 10.1016/S01406736(00)87996-9

Fukuhara, S., Sakurai, A., Sano, H., Yamagishi, A., Somekawa, S., Takakura, N., et al. (2005). Cyclic AMP potentiates vascular endothelial cadherin-mediated cell-cell contact to enhance endothelial barrier function through an Epac-Rap1 signaling pathway. Mol. Cell. Biol. 25, 136-146. doi: 10.1128/MCB.25.1.136146.2005

Gonzales, J. N., Gorshkov, B., Varn, M. N., Zemskova, M. A., Zemskov, E. A., Sridhar, S., et al. (2014). Protective effect of adenosine receptors against lipopolysaccharide-induced acute lung injury. Am. J. Physiol. Lung Cell. Mol. Physiol. 306, L497-L507. doi: 10.1152/ajplung.00086.2013

Herwig, M. C., Tsokos, M., Hermanns, M. I., Kirkpatrick, C. J., and Müller, A. M. (2013). Vascular endothelial cadherin expression in lung specimens of patients with sepsis-induced acute respiratory distress syndrome and endothelial cell cultures. Pathobiology 80, 245-251. doi: 10.1159/000347062

Izdebski, J., Pinski, J., Horvath, J. E., Halmos, G., Groot, K., and Schally, A. V. (1995). Synthesis and biological evaluation of superactive agonists of growth hormone-releasing hormone. Proc. Natl. Acad. Sci. U.S.A. 92, 4872-4876. doi: 10.1073/pnas.92.11.4872

Kanashiro-Takeuchi, R. M., Tziomalos, K., Takeuchi, L. M., Treuer, A. V., Lamirault, G., Dulce, R., et al. (2010). Cardioprotective effects of growth hormonereleasing hormone agonist after myocardial infarction. Proc. Natl. Acad. Sci. U.S.A. 107, 2604-2609. doi: 10.1073/pnas.0914138107

Kiaris, H., Chatzistamou, I., Papavassiliou, A. G., and Schally, A. V. (2011). Growth hormone-releasing hormone: not only a neurohormone. Trends Endocrinol. Metab. 22, 311-317. doi: 10.1016/j.tem.2011.03.006

Lania, A., Filopanti, M., Corbetta, S., Losa, M., Ballaré, E., Beck-Peccoz, P., et al. (2003). Effects of hypothalamic neuropeptides on extracellular signal-regulated kinase (ERK1 and ERK2) cascade in human tumoral pituitary cells. J. Clin. Endocrinol. Metab. 88, 1692-1696. doi: 10.1210/jc.2002-021207

Lawrence, D. W., Comerford, K. M., and Colgan, S. P. (2002). Role of VASP in reestablishment of epithelial tight junction assembly after $\mathrm{Ca}^{2+}$ switch. Am. J. Physiol. Cell Physiol. 282, C1235-C1245. 10.1152/ajpcell.00288.2001

Lucas, R., Czikora, I., Sridhar, S., Zemskov, E. A., Oseghale, A., Circo, S., et al. (2013). Arginase 1: an unexpected mediator of pulmonary capillary barrier dysfunction in models of acute lung injury. Front. Immunol. 4:228. doi: 10.3389/fimmu. 2013.00228

Lucas, R., Sridhar, S., Rick, F. C., Gorshkov, B., Umapathy, N. S., Yang, G., et al. (2012a). Agonist of growth hormone-releasing hormone reduces pneumolysin-induced pulmonary permeability edema. Proc. Natl. Acad. Sci. U.S.A. 109, 2084-2089. doi: 10.1073/pnas.1121075109

Lucas, R., Verin, A. D., Black, S. M., and Catravas, J. D. (2009). Regulators of endothelial and epithelial barrier integrity and function in acute lung injury. Biochem. Pharmacol. 77, 1763-1772. doi: 10.1016/j.bcp.2009. 01.014

Lucas, R., Yang, G., Gorshkov, B. A., Zemskov, E. A., Sridhar, S., Umapathy, N. S., et al. (2012b). Protein kinase C-alpha and arginase I mediate pneumolysininduced pulmonary endothelial hyperpermeability. Am. J. Respir. Cell Mol. Biol. 47, 445-453. doi: 10.1165/rcmb.2011-0332OC

Ludwig, B., Rotem, A., Schmid, J., Weir, G. C., Colton, C. K., Brendel, M. D., et al. (2012). Improvement of islet function in a bioartificial pancreas by enhanced oxygen supply and growth hormone releasing hormone agonist. Proc. Natl. Acad. Sci. U.S.A. 109, 5022-5027. doi: 10.1073/pnas.12018 68109

Maus, U. A., Srivastava, M., Paton, J. C., Mack, M., Everhart, M. B., Blackwell, T. S., et al. (2004). Pneumolysin-induced lung injury is independent of leukocyte trafficking into the alveolar space. J. Immunol. 173, 1307-1312. doi: 10.4049/jimmunol.173.2.1307

Mayo, K. E., Godfrey, P. A., Suhr, S. T., Kulik, D. J., and Rahal, J. O. (1995). Growth hormone-releasing hormone: synthesis and signaling. Recent Prog. Horm. Res. $50,35-73$.

Moretti, C., Mencacci, C., Frajese, G. V., Cerilli, M., and Frajese, G. (2002). Growth hormone-releasing hormone and pituitary adenylate cyclase-activating polypeptide in the reproductive system. Trends Endocrinol. Metab. 13, 428-435. doi: 10.1016/S1043-2760(02)00632-X

Petrache, I., Birukova, A., Ramirez, S. I., Garcia, J. G., and Verin, A. D. (2003). The role of the microtubules in tumor necrosis factor-alpha-induced endothelial cell permeability. Am. J. Resp. Cell. Mol. Biol. 28, 574-581. doi: 10.1165/rcmb.20020075OC

Qiao, J., Holian, O., Lee, B. S., Huang, F., Zhang, J., and Lum, H. (2008). Phosphorylation of GTP dissociation inhibitor by PKA negatively regulates RhoA. Am. J. Physiol. Cell Physiol. 295, C1161-C1168. doi: 10.1152/ajpcell.00139.2008

Rafikov, R., Dimitropoulou, C., Aggarwal, S., Kangath, A., Gross, C., Pardo, D., et al. (2014). Lipopolysaccharide-induced lung injury involves the nitration-mediated activation of RhoA. J. Biol. Chem. 289, 4710-4722. doi: 10.1074/jbc.M114.547596

Rekasi, Z., Czompoly, T., Schally, A. V., and Halmos, G. (2000). Isolation and sequencing of cDNAs for splice variants of growth hormone-releasing hormone receptors from human cancers. Proc. Natl. Acad. Sci. U.S.A. 97, 10561-10566. doi: $10.1073 /$ pnas. 180313297

Rubins, J. B., Charboneau, D., Fasching, C., Berry, A. M., Paton, J. C., Alexander, J. E., et al. (1996). Distinct roles for pneumolysin's cytotoxic and complement activities in the pathogenesis of pneumococcal pneumonia. Am. J. Respir. Crit. Care Med. 153, 1339-1346. doi: 10.1164/ajrccm.153.4.86 16564

Rudan, I., Boschi-Pinto, C., Biloglav, Z., Mulholland, K., and Campbell, H. (2008). Epidemiology and etiology of childhood pneumonia. Bull. World Health Organ. 86, 408-416. doi: 10.2471/BLT.07.048769

Schlegel, N., Baumer, Y., Drenckhahn, D., and Waschke, J. (2009). Lipopolysaccharide-induced endothelial barrier breakdown is cyclic adenosine monophosphate dependent in vivo and in vitro. Crit. Care Med. 37, 1735-1743. doi: 10.1097/CCM.0b013e31819deb6a

Sherwood, N. M., Krueckl, S. L., and McRory, J. E. (2000). The origin and function of the pituitary adenylate cyclase activating polypeptide (PACAP)/glucagon superfamily. Endocr. Rev. 21, 619-670. doi: 10.1210/edrv.21. 6.0414

Umapathy, N. S., Gonzales, J., Fulzele, S., Kim, K. M., Lucas, R., and Verin, A. D. (2012). $\beta$-Nicotinamide adenine dinucleotide attenuates lipopolysaccharide-induced inflammatory effects in a murine model of acute lung injury. Exp. Lung Res. 38, 223-232. doi: 10.3109/01902148.2012. 673049

Umapathy, N. S., Zemskov, E. A., Gonzales, J., Gorshkov, B. A., Sridhar, S., Chakraborty, T., et al. (2010). Extracellular beta-nicotinamide adenine dinucleotide (beta-NAD) promotes the endothelial cell barrier integrity via PKA- and EPAC1/Rac1-dependent actin cytoskeleton rearrangement. J. Cell. Physiol. 223, 215-223. doi: 10.1002/jcp. 22029 
Waterer, G. W., Rello, J., and Wunderink, R. G. (2011). Management of communityacquired pneumonia in adults. Am. J. Respir. Crit. Care Med. 183, 157-164. doi: 10.1164/rccm.201002-0272CI

Witzenrath, M., Gutbier, B., Hocke, A. C., Schmeck, B., Hippenstiel, S., Berger, K., et al. (2006). Role of pneumolysin for the development of acute lung injury in pneumococcal pneumonia. Crit. Care Med. 34, 1947-1954. doi: 10.1097/01.CCM.0000220496.48295.A9

Zhao, Y., Zhao, J., Mialki, R. K., Wei, J., Spannhake, E. W., Salgia, R., et al. (2013). Lipopolysaccharide-induced phosphorylation of c-Met tyrosine residue 1003 regulates c-Met intracellular trafficking and lung epithelial barrier function. Am. J. Physiol. Lung Cell. Mol. Physiol. 305, L56-L63. doi: 10.1152/ajplung.00417.2012

Conflict of Interest Statement: The authors declare that the research was conducted in the absence of any commercial or financial relationships that could be construed as a potential conflict of interest.
Received: 12 April 2014; accepted: 19 June 2014; published online: 15 July 2014. Citation: Czikora I, Sridhar S, Gorshkov B, Alieva IB, Kasa A, Gonzales J, Potapenko O, Umapathy NS, Pillich H, Rick FG, Block NL, Verin AD, Chakraborty T, Matthay MA, Schally AV and Lucas R (2014) Protective effect of Growth Hormone-Releasing Hormone agonist in bacterial toxin-induced pulmonary barrier dysfunction. Front. Physiol. 5:259. doi: 10.3389/fphys.2014.00259

This article was submitted to Oxidant Physiology, a section of the journal Frontiers in Physiology.

Copyright (C) 2014 Czikora, Sridhar, Gorshkov, Alieva, Kasa, Gonzales, Potapenko, Umapathy, Pillich, Rick, Block, Verin, Chakraborty, Matthay, Schally and Lucas. This is an open-access article distributed under the terms of the Creative Commons Attribution License (CC BY). The use, distribution or reproduction in other forums is permitted, provided the original author(s) or licensor are credited and that the original publication in this journal is cited, in accordance with accepted academic practice. No use, distribution or reproduction is permitted which does not comply with these terms. 